\title{
Behavioral Effects of Sequential and One-Stage Ablations of Orbital Prefrontal Cortex in the Monkey
}

\author{
NELSON ButTers ${ }^{1}$ \\ Psychology Service, Boston Veterans Administration Hospital, and Aphasia Research \\ Center, Neurology Dept., Boston University School of Medicine, \\ Boston, Massachusetts 02130
}

Charles Butter

Psychology Department, University of Michigan, Ann Arbor, Michigan 48104

JEFFREY Rosen

Psychology Department, Boston University, Boston, Massachusetts 02215

Donald Stein

Psychology Department, Clark University. Worcester, Massachusetts 01610

Received September 16, 1972

\begin{abstract}
The purpose of this investigation was to determine whether sequential (i.e., serial) ablation of the monkey's orbital prefrontal cortex would lead to a reduction in the severity of the behavioral impairment usually associated with one-stage bilateral removal of this tissue. The lateral orbital cortex was ablated in four operations spaced 3 weeks apart or in a one-stage procedure. The monkeys were examined on a visual go-no go differentiation task, spatial delayed-alternation, and object reversal learning. The results reveal no differences between the effects of sequential and one-stage ablations. These findings differ from previous experiments that demonstrated a degree of functional recovery after the sequential removal of a sector of the dorsolateral prefrontal cortex. Since lesion studies with infant monkeys have also demonstrated that functional recovery occurs after early ablation of dorsolateral cortex but not after early removal of orbital frontal cortex, recovery of behavioral functions after infant and sequential lesions may involve similar neural mechanisms.
\end{abstract}

\section{INTRODUCTION}

Recovery of function in primates after extensive brain ablations occurs most often when the ablation is performed on infant animals or when

${ }^{1}$ Supported in part by N.I.II. grants NS-06209 to Boston University and NS-08606 to Clark University. 
the lesion is produced sequentially (i.e., multiple stages) in the adult. For example, ablation of the dorsolateral prefrontal cortex in the infant monkey results in attenuated delayed-response deficits $(6,9,17)$, and sequential ablation of sulcus principalis (on the dorsolateral prefrontal surface) in mature monkeys produces less severe spatial deficits on delayed-response and alternation than does a one-stage bilateral ablation $(4,16)$.

These generalizations, however, do not hold for all parts of the brain. Functional recovery occurs after lesions of the clorsolateral prefrontal cortex in infant monkeys, but not after equally early lesions of the orbital prefrontal cortex $(6-8)$. Given this functional dissociation after early dorsolateral and orbital lesions. it seems appropriate to determine whether sequential ablation of the orbital cortex in the mature monkey will result in recovery of function. If recovery of function does not occur following sequential orbital lesions, this would provide further evidence that the recovery phenomenon is not characteristic of all cortical structures. In addition, although the neural mechanisms underlying behavioral recovery are not well understood, the finding of sinilar patterns of recovery after early and sequential lesions would strengthen the possibility that similar mechanisms are involved in both types of behavioral sparing.

In the present experiment, monkeys with one-stage or sequential bilateral ablations of the lateral orbital cortex were compared on three tasks on which the behavior of the animals is known to be impaired after one-stage bilateral orbital lesions $(2,13)$. Unoperated control monkeys were also examined on the same tasks.

\section{METHODS}

Fifteen naive rhesus monkeys (Macaca mulatta) weighing 3-4 kg served as subjects; they were housed individually and maintained on partial food deprivation schedules. Fourteen of the 15 monkeys completed all behavioral testing but one unoperater control animal became ill after delayed-alternation testing and did not complete the object reversal task.

Beharioral Testing. The monkeys were tested individually in a Wisconsin General Test Apparatus which employed a vertically sliding opaque screen to separate the monkey compartment from the test trays and stimuli. Three tests were given; go-no go differentiation $(\mathrm{G}-\mathrm{NG})$; spatial delayed-alternation (DA); and object reversal learning (OR).

Preoperatively, all monkeys learned a visual G-NG problem. On each trial the monkeys were presented with a tray containing a single foodwell. On half the trials, the food well was covered by a gray cardboard plaque on which a white outlined paper square had heen glued; on the remaining trials, the figure on the gray plaque was a white plus sign. If the monkeys displaced the plus plaque within $5 \mathrm{sec}$ after exposure, they were allowed to 
retrieve a raisin from the food well and were credited with a correct response. If the monkeys failed to respond to the plus plaque within $5 \mathrm{sec}$, the screen was lowered between the monkeys and the tray, the monkeys received no reinforcement, and they were credited with an error. If the monkeys displaced the square plaque within $5 \mathrm{sec}$, they received no reinforcement and were credited with an error. If the monkeys refrained from displacing the square plaque for $5 \mathrm{sec}$, the screen was lowered, and they received no reward but were credited with a correct response. Thus, the monkeys had to learn to respond to the plus and not to respond to the square. Thirty noncorrection trials were administered daily until the monkeys reached a criterion of at least 90 correct responses in 100 consecutive trials. After attaining this criterion the monkeys were assigned to one of the operated or control groups. Two weeks following their last operation (or after 10 weeks of rest for the unoperated controls), the monkeys were tested for G-NG retention. The same procedures and learning criterion employed preoperatively were used again.

After completion of G-NG retention, the monkeys were trained on the acquisition of 5-sec spatial DA. On each trial the monkeys were confronter with a tray containing two food wells, one on the right and one on the left, covered by identical black wooden plaques. Briefly, the monkeys had to learn to alternate their spatial responses (right-left) on successive trials which were separated by a 5 -sec delay. During the delay period the opaque screen separated the monkeys from the testing tray. On the first trial of cach test session both food wells contained a reward (raisins were used as reinforcements). A rerun correction procedure was employed: If the monkeys made an error on any given trial, the reinforcement remained on the same side on succeeding trials until the monkcys made a correct response. Thirty trials, including correction trials, were administered each day until the monkeys reached a criterion of at least 90 correct responses in 100 conseculive trials. If a monkey failed to learn within 1000 trials, testing was terminated.

Upon completion of DA testing, all monkeys were trained on an object reversal task (OR). On each trial the monkeys were presented with two food wells covered by wooden objects (glued to wooden plaques) differing in color, shape, and size and were allowed to displace one of the plaques. If the monkeys chose the correct object, they received a raisin reward. Initially, the monkeys were trained to respond to one of the two objects. When the monkeys reached criterion on the original discrimination (at least 27 correct responses on 30 consecutive trials), the reward value of the ol)jects was switched (reversal 1) and the monkeys had to learn this reversal to criterion. Five successive reversals were learned by all monkeys. Thirty trials were administered each day. The same pair of objects was used for 
each of the animals. The object reinforced on any given discrimination or reversal remained constant for all subjects.

Surgical Procedutes. In all operations, the monkeys were first immobilized with Sernylan (1.5 $\mathrm{mg} / \mathrm{kg}$, im) followed by sodium pentobarbital (15 mg/kg, iv). Using sterile precautions, the scalp, fascia, and temporal muscles were incised and retracted, and skull openings were made bilaterally over the prefrontal area. The dura was then incised and reflected, and lateral orbital cortex was removed by subpial aspiration with a narrow-gauge sucker. The intended area of the lesion included all cortex on the convexity separating the dorsolateral and orbital prefrontal cortex. The intended boundary dorsally was a horizontal line $5 \mathrm{~mm}$ below sulcus principalis and ventrally, the lateral orbital sulcus. Anteriorly the boundary of the intended lesion was the frontal pole. The posterior boundary was the anterior bank of the descending limb of the arcuate sulcus on the lateral surface, and on the ventral surface the intended lesion involved all lateral orbital cortex. Bleeding was controlled by packing with Cottonoid pads, and the wound was closed in anatomical layers with silk sutures. At the completion of surgery, 600,000 units of penicillin were administered intramuscularly.

The five monkeys assigned to the one-stage lesion group had a single bilateral operation 10 weeks after reaching criterion. The five monkeys assigned to the sequential (i.e., serial) lesion group received four operations spaced 3 weeks apart. For each operation the hemisphere and sector of the lateral orbital cortex were switched. In the first operation the lateral orbital cortex of the left hemisphere was removed; in the second, the inferior dorsolateral surface of the right hemisphere: in the third, the inferior dorsolateral surface of the left hemisphere; and finally, the lateral orbital surface of the right hemisphere was removed in the fourth operation.

Histological Proccdures. After all testing was completed, the operated monkeys were deeply anesthetized with sodium pentobarbital and perfused intracardially with $0.9 \%$ saline solution followed by $10 \%$ formalin. Their brains were then removed, embedded in alhumin-gel, and frozen. Sections $40 \mu \mathrm{m}$ in thickness were cut through the area of the lesion. Every tenth section was mounted and stained with cresyl violet for histological examination. The extent of the lesion in each section was measured with calipers and transferred to a diagrammatic model of the orbital and dorsolateral frontal cortex. A polar planimeter was used to compute the total area of damage. With these measurements it was possible to compare the size of the lesions in the two operated groups.

\section{RESUTTS}

Histological Analysis. Reconstruction of the serial and one-stage lesions are shown in Fig. 1. In general, the lesions included all the lateral orbital 

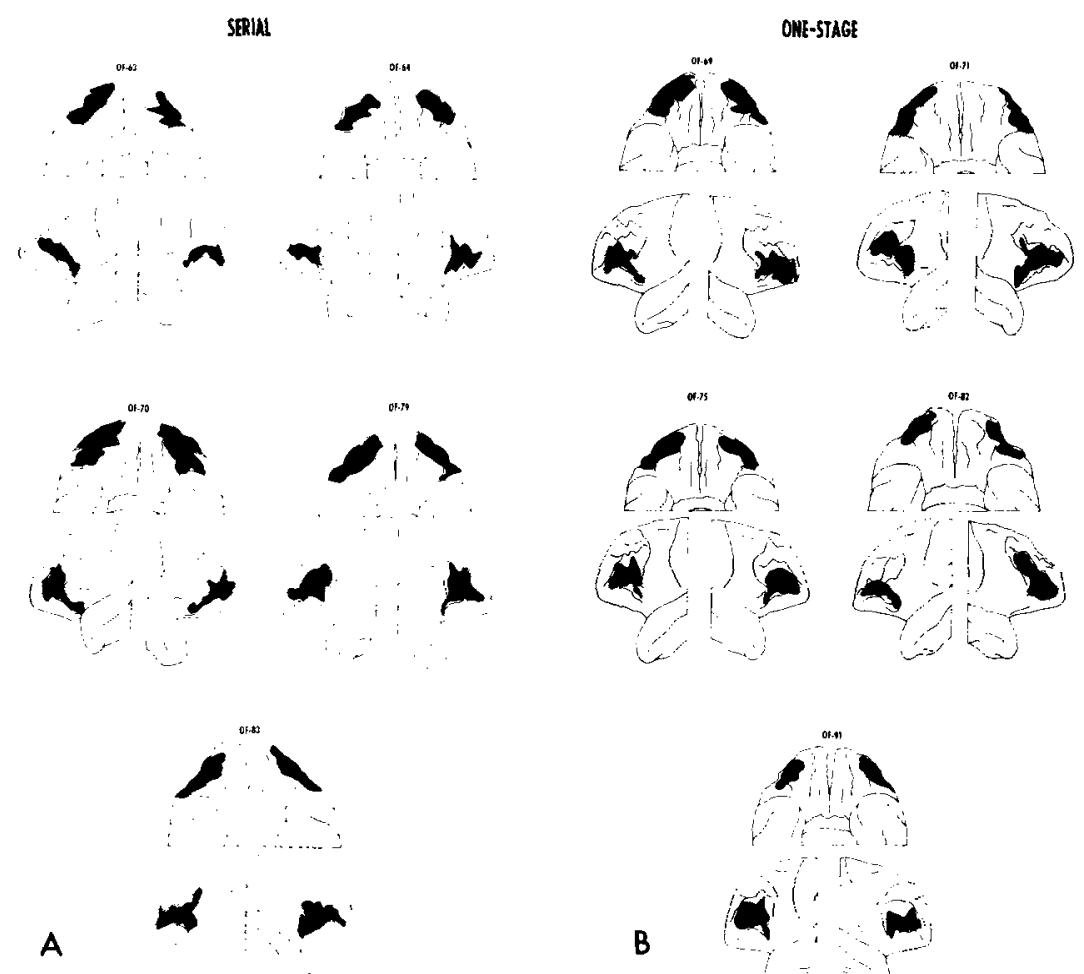

FIg. 1. (A) Ventral and lateral views of the serial lateral orbital frontal lesions (in black). (B) Ventral and lateral views of the one-stage lateral orbital frontal (in black).

cortex intended to be removed, although several of the animals in each operated group had lesions extending up to sulcus principalis and involving to varying degrees the lower bank of this sulcus. In the monkeys with serial lesions this dorsal extension was most likely due to adhesions between the dura and pia mater that formed after the first operation. In order to make lesions equivalent in the two operated groups, the ventral bank of sulcus principalis was deliberately removed in three monkeys of the one-stage group. As a result, the lesions sustained by the two operated groups did not differ in extent either on the dorsolateral or orbital surface, as indicated by planimetric measurements. Moreover, the lesions of animals in both operated groups were restricted to the cortex, with the exception of unilateral involvement of the claustrum in one monkey with serial lesions.

Go-No Go Performance. The Fisher randomization test (16) was employed for all statistical analyses in this study. One-tailed probabilities were employed when either of the surgical groups' performance was compared with the control group. Two-tailed probabilities were used in con- 
trasting the performance of the two operated groups. However, since no differences were anticipated on $\mathrm{G}-\mathrm{NG}$ accuisition or on the initial object discrimination, two-tailed probabilities were employed for all group comparisons on these two tasks.

All monkeys learned G-NG preoperatively, and the three groups did not differ significantly in the number of errors required to attain criterion (control $=354$ mean errors; sequential $=333$ mean errors; one-stage $=$ 276 mean errors).

Analyses of the monkeys' postoperative performance (Fig. 2A) on G-NG showed significant differences among the groups. Both the sequential and one-stage groups made significantly more errors than did the control group $(p=0.007)$. The difference between the one-stage and serial groups did not approach significance $(p>0.40)$.

Delayed Alteration Acquisition. The mean number of errors required by the three groups to learn DA are prescnted in Fig. 2B. The control group made fewer errors than did the one-stage and sequential groups $(p=0.05$, $p=0.10$, respectively). The difference between the sequential and one-stage groups did not approach significance $(p>0.40)$.

The sequential vs. control and the one-stage vs. unoperated control comparisons did not attain higher levels of significance because one monkey in the control group failed to learn DA within 1000 trials. Since this animal is the only intact monkey to fail DA acquisition in the first author's laboratory during the past 5 years, its DA error score (279 errors) is not representative of normal DA performance. If this animal is excluded from DA analyses, the mean error score of the controls drops to 133, and the comparisons between the control and the two operated groups are both highly significant (one-stage vs. moperated control, $p=0.028$; sequential vs. unoperated control, $p=0.034$ ).
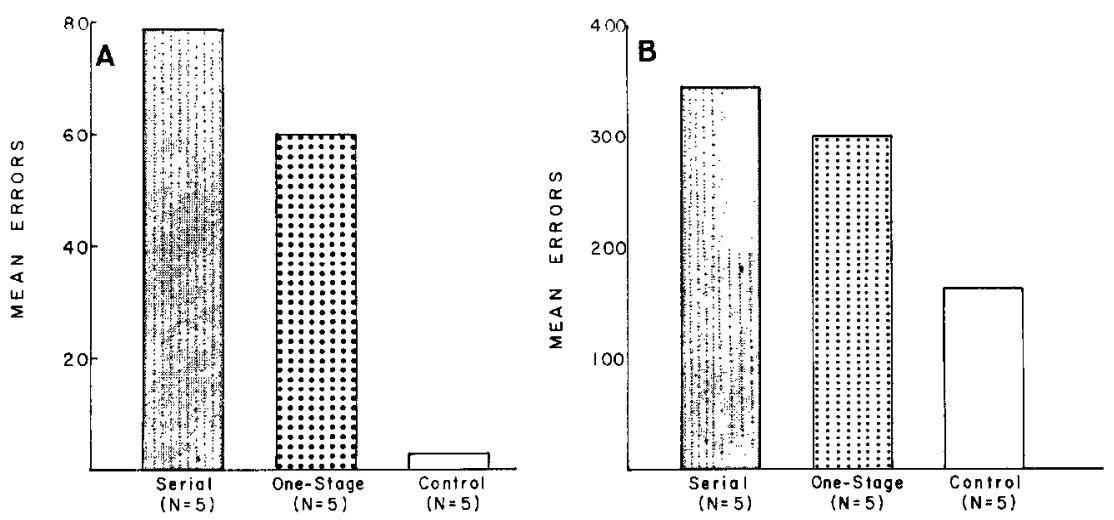

FIG. 2. (A) Mean errors in the retention of the G-NG task. (B) Mean errors in the acquisition of 5 -sec spatial DA. 


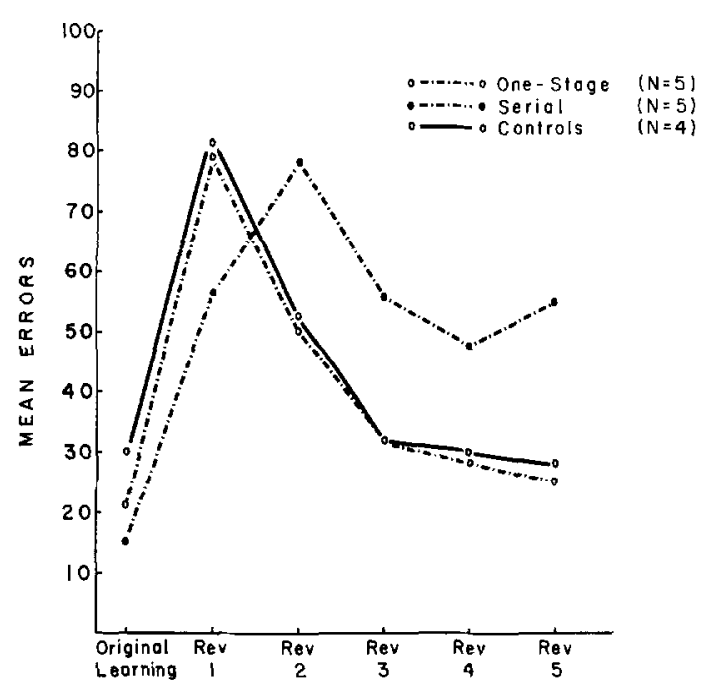

Fia. 3. Mean errors on OR learting.

Object Reversal Performance. Figure 3 shows the mean number of errors compiled by the three groups on the initial object discrimination and the five reversals. On the initial object discrimination, both the one-stage and sequential groups made significantly $(p=0.032, p=0.008$, respectively) fewer errors than did the controi group. On the first reversal, the one-stage and normal control groups made more errors than they had on the original discrimination, but on each succeeding reversal (2-5) the two groups showed a decrement in their error scores (i.e., a reversal learning set). In contrast, the monkeys with serial ablations did not evidence the same degree of successive improvement. These monkeys made more mean errors on the second, third, and fifth reversals than they had on the first reversal. To assess these different reversal learning functions, the number of errors on the fifth reversal was subtracted from the number of errors on the first reversal. Table 1 presents the mean errors on the first and fifth reversals and the mean reversal improvement score (Rev. 1-Rev. 5). A positive rcversal improvcment score indicatcs that the monkeys made fewer errors on the fifth than on the first reversal (i.e., the establishment of a reversal learning set); a negative reversal improvement score indicates that more errors were made on the fifth than on the first reversal (i.e., a failure to establish a reversal learning set).

While the sequential group had a negative reversal improvement score, both the control and one-stage groups had positive scores. The sequential vs. one-stage and the sequential vs. control comparisons were both significant ( $p=0.012, p=0.001$, respectively). The comparison of the one-stage and control improvement scores did not approach significance $(p>0.40)$. 
TABLE 1

Object Reversal: Mean Erroks on Initial Object Discrimination and on Reversals 1 and 5 and Reversal Improvement Scores

\begin{tabular}{cccccc}
$\begin{array}{c}\text { No. of } \\
\text { monkeys }^{a}\end{array}$ & $\begin{array}{c}\text { Initial } \\
\text { discrimin. }\end{array}$ & $\begin{array}{c}\text { Rev. } \\
1\end{array}$ & $\begin{array}{c}\text { Rev. } \\
5\end{array}$ & $\begin{array}{l}\text { Rev. } \\
1-5\end{array}$ & $\frac{\text { Init. - Rev. 5 }}{\text { Init. }}$ \\
\hline 5 SS & 14 & 55 & 56 & -1 & -3.00 \\
5 OS & 20 & 78 & 25 & +53 & -0.25 \\
4 NC & 34 & 82 & 27 & +55 & +0.20 \\
\hline
\end{tabular}

a Abbreviations: SS, serial operations; OS, one-stage operations; NC, normal controls.

The apparently normal reversal perfornance of the one-stage group was surprising because of the many reports (e.g., 2, 13) demonstrating object reversal deficits after one-stage bilateral orbital lesions. Since the one-stage group was superior to the control group on the original discrimination, the apparent normality of the one-stage group on OR might have reflected a failure to consider initial group differences in acquiring object discriminations. To assess this possibility, a second reversal improvement score was derived: errors on the fifth reversal were subtracted from errors on the initial object discrimination and this difference was divided by errors on the initial object discrimination.

Table 1 shows the mean errors for the three groups on the initial object discrimination and on the fifth reversal as well as the reversal improvement score (Init. Disc. - Rev. 5/Init. Disc. ). While the control group had a positive reversal improvement score, both the sequential and the one-stage groups had negative scores. All group comparisons now attained statistical significance (sequential vs. control, $p=0.015$; one-stage vs. control, $p=$ 0.032 ; sequential vs. one-stage, $p=0.024$ ).

Spatial Alternation during Object Discrimination Learning. When the monkeys were being tested on the original object discrimination, it appeared to the examiner that the control group was impaired on this task because they continued to perform spatial alteration (the previous task) for the first 30 trials of discrimination training. Since most of the monkeys with sequential or one-stage lesions either failed to learn DA or were severely impaired on this spatial task, they demonstrated little transfer (negative) of DA habits to the object discrimination task.

To quantify and statistically analyze these observations, a measure of spatial alternation during the first 30 trials of object discrimination training was developed. For each monkey, a theoretical spatial alternation sequence was generated on the basis of the animal's spatial response on the first trial of the 30 trial session. For example, if the animal responded to the object on the right on the first trial, its theoretical spatial alternation performance 
for the first ten trials was $R, L, R, L, R, L, R, L, R, L$. This theoretical sequence which assumes perfect alternation was compared with the aninal's actual spatial responses on each of the 30 trials, and the number of trials on which the theoretical and actual spatial responses corresponded was noted. Thus, if the previous monkey's actual response pattern was $R, R, L$, $L, R, L, R, L, L, L$, its spatial alternation score for the first ten trials would be seven (the theoretical and actual spatial responses correspond except for the second, third, and ninth trials).

The control group (mean $=19.75$ ) displayed more spatial alternation during the first 30 trials of object discrimination training than did the onestage $($ mean $=15.40$ ) or sequential (mean $=13.40$ ) groups. The difference between the control and one-stage groups was significant $(p=0.045)$; the difference between the control and sequential groups was also significant $(p=0.04)$; the difference between the sequential and one-stage groups did not approach significance $(p>0.40)$.

\section{DISCUSSION}

The present results indicate that sequential ablation of the lateral orbital cortex does not reduce the severity of the behavioral dysfunction usually associated with lesions produced in one-stage. On go-no go retention and spatial delayed-alternation, both the one-stage and sequential groups were impaired in comparison to the normal control group. Comparisons of the sequential and one-stage groups on these tests showed that the monkeys with sequential lesions made slightly, but not significantly, more errors than did the one-stage monkeys. On object-reversal learning the one-stage and normal control groups both showed evidence of reversal learning set formation (i.e., successive improvement in the learning of the five reversals), but the sequentially operated group failed to show such a trend. When we employed a measure of reversal learning that took into account initial differences among the group in object discrimination learning, the one-stage, as well as the sequential, group was impaired significantly in comparison to the normal control group.

These findings for sequential orbital lesions contrast with the results of previous investigations concerned with recovery of function after serial ablations of dorsolateral prefrontal cortex $(4,16)$. When sulcus principalis is ablated in four operations spaced three weeks apart, monkeys show considerable recovery of behavioral functions (i.c., spatial delayed-alternation, delayed-response, place reversal learning) impaired when this region is ablated in one stage.

This dissociation between the effects of sequential dursolateral and orbital prefrontal ablations indicates that recovery of function is not characteristic of all sequentially ablated cortical tissue and suggests at least one hypothesis 
with regard to the limitations of the recovery phenomenon. In addition to their capacities for behavioral recovery, the dorsolateral and orbital cortices also subserve different behavioral functions and are associated with different anatomical structures. The dorsolateral cortex is concerned with spatial and mnemonic functions $(5,13)$ and has strong connections with the basal ganglia (e.g., anterodorsal sector of the head of the caudate nucleus), cingulate cortex, and temporal neocortex $(10,14,15)$. The orbital cortex is involved in response or drive inhibition. or both $(1,2,13)$, as well as the modulation of aversive and aggressive bchaviors (3) and has, in contrast to the dorsolateral sector, many connections with limbic structures like the amygdala, septal nuclei, and hypothalamus $(10,14)$. These anatomical and behavioral differences suggest that, in the primate, recovery of function may follow sequential or early ablation of structures with sensory (12), motor (11) and associative functions but may be more limited for neural structures concerned with motivational processes.

Finally, the present results closely parallel the findings from early lesion studies (6-8). Goldman has demonstrated that recovery of function occurs (10 months after surgery) after removal of dorsolateral prefrontal cortex in infancy but not after equally early ablation of the orbital prefrontal region. These similarities in the patterns of recovery after sequential and early lesions suggest that similar neural mechanisms may be mediating both types of behavioral recovery. More recently Goldman $(7,8)$ has reported that monkeys that had their dorsolateral cortex removed in infancy (within 30 days of birth) and had evidenced behavioral recovery 10 months following surgery were severely impaired when tested at 2 years of age; conversely, monkeys that had orbital lesions during infancy and had shown little, if any, recovery 10 months after surgery displayed considerable recovery when they were tested at 2 years of age $(7,8)$. According to Goldman's interpretation of this finding. the clorsolateral and orbital frontal cortices show differential rates of anatomical development. If this interpretation is valid, it would suggest that recovery of function following sequential lesions of a neural structure in adulthood is related to the rate of anatomical development of that structure in infancy.

\section{REFERENCES}

1. Brutkowski, S. 1964. Prefrontal cortex and drive inhibition, pp. 242-270. In "The Frontal Granular Cortex and Behavior." J. M. Warren and K. Akert [Eds.]. McGraw-Hill, New York.

2. Butrer, C. M. 1969. Perseveration in extinction and in diserimination reversal tasks following sefective frontal ablations in Macaca Mulatta. Physiol. Bchare 4: 163-171.

3. ButTer, C. M., and D. R. SNymer. 1972. Alterations in aversive and aggressive behaviors following orbital frontal lesions in rhesus monkeys. Acta Biol. Exp. 32: $525-566$. 
4. Butters, N., D. Pandya, D. Stein, and J. Rosen. 1972. A search for the spatial engram within the frontal lobes of monkeys. Acta Biol. Exp. 32: 305-330.

5. Goldman, P. S., and H. E. Rosvold. 1970. Localization of function within the dorsolateral prefrontal cortex of the rhesus monkey. Exp. Neurol. 27: 291-304.

6. Goldman, P., H. E. Rosvor.d, and M. Mishkin, 1970. Evidence for behavioral impairment following prefrontal lobectomy in the infant monkey. J. Comp. Physiol. Psychol. 70: 454463.

7. Goldman, P. 1971. Functional development of the prefrontal cortex in early life and the problem of neuronal plasticity. Exp. Neurol. $32: 366-387$.

8. Goldman, P. 1972. Developmental determinants of cortical plasticity. Acta Biol. Exp. 32: 495-512.

9. Harlow, H. F., A. K. Akert, and K. A. Schiltz. 1964. The effects of bilateral prefrontal lesions on learned behavior of neonatal, infant, and pre-adolescent monkeys, pp. 126-148. In "The Frontal Granular Cortex and Behavior." J. M. Warren and K. Akert [Eds.]. McGraw-Hill, New York.

10. Johnson, T. N., H. E. Rosvold, and M. Mrshirin. 1968. Projections of behaviorally-defined sectors of the prefrontal cortex to the basal ganglia, septum, and diencephalon of the monkey. Exp. Neurol. 21:20-34.

11. Kennard, M. A. 1938. Reorganization of motor function in the cerebral cortex of monkeys deprived of motor and premotor areas in infancy. J. Neurophysiol. $1: 477-496$.

12. Meyer, D. R. 1958. Some psychological determinants of sparing and loss following damage to the brain, pp. 173-192. In "Biological and Biochemical Bases of Behavior." H. F. Harlow and C. N. Woolsey [Eds.]. University of Wisconsin Press, Madison.

13. Mishkin, M. 1964. Perseveration of central sets after frontal lesions in monkeys, pp. 219-241. In "The Frontal Granular Cortex and Behavior." J. M. Warren and K. Akert [Eds.]. McGraw-Hill, New York.

14. Nauta, W. J. H. 1964. Some efferent connections of the prefrontal cortex in the monkey, pp. 397-409. In "The Frontal Granular Cortex and Behavior." J. M. Warren and K. Akert [Eds.]. McGraw-Hill, New York.

15. Pandya, D. N., P. Dye, and N. ButTers. 1971. Efferent cortico-cortical projections of the prefrontal cortex in the rhesus monkey. Brain Res. 31:21-34.

16. Rosen, J., D. Stein, and N. Butters. 1971. Recovery of function after serial ablation of prefrontal cortex in the rhesus monkey. Science 173: 353-356.

17. TuCker, T. J., and A. KLING. 1969. Preservation of delayed response following combined lesions of prefrontal and posterior association cortex in infant monkeys. Exp. Neurol. 23 : 491-502. 